\title{
Juvenile idiopathic arthritis associated uveitis
}

\author{
Ying Qian, M.D. ${ }^{1,2}$ and Nisha R. Acharya, M.D., M.S. ${ }^{1,2}$ \\ ${ }^{1}$ F.I. Proctor Foundation, University of California, San Francisco, California, USA \\ 2 Department of Ophthalmology, University of California, San Francisco, California, USA
}

\section{Abstract}

Purpose of Review-To describe recent evidence from the literature pertaining to juvenile idiopathic arthritis (JIA) associated uveitis.

\begin{abstract}
Recent Findings-Uveitis is most common in extended oligoarticular JIA. A significant number of patients already have ocular complications at time of diagnosis of uveitis. Risk factors for complications include either abnormally high or low intraocular pressure, posterior synechiae, male gender, temporal proximity to diagnosis of arthritis and topical corticosteroid use. Use of immunosuppressive agents significantly reduces ocular complications. Aggressive peri-operative control of intraocular inflammation is necessary for successful cataract surgery with lens implantation. Controlled clinical trials are under way to assess the efficacy of biologic agents in JIA-associated uveitis. Long term safety, however, is still unknown.
\end{abstract}

Summary-JIA-associated uveitis carries significant ocular morbidity that lasts well into adulthood. Treatment with immunosuppressive agents can reduce the risk of ocular complications. Biologics hold promise in the treatment of JIA-associated uveitis but require long-term data to assess their safety.

\section{Keywords}

juvenile idiopathic arthritis; uveitis; immunomodulatory therapy; immunosuppression

\section{Introduction}

Uveitis is observed in 30\% of ANA positive patients with juvenile idiopathic arthritis (JIA) [1] and is an important cause of ocular morbidity in childhood and beyond. The use of immunosuppressive therapy has greatly reduced the ocular complications from JIAassociated uveitis [2]. Tight perioperative control of intraocular inflammation has also enabled successful cataract surgery to be performed, with implantation of intraocular lenses. While biologic agents hold promise in the treatment of JIA-associated uveitis, their longterm safety profile has yet to be established.

\section{JIA - Epidemiology and Pathogenesis}

JIA is a collection of heterogeneous chronic childhood arthritides with onset before age 16 and persisting 6 weeks or longer with likely distinct pathophysiologic mechanisms that lead to a common pattern of tissue destruction [3]. There are four subtypes of JIA: persistent oligoarticular (4 or less joints involved throughout course of disease), extended

Correspondence to: Nisha Acharya, MD, MS., F.I. Proctor Foundation, Room S309, 513 Parnassus Avenue, University of California San Francisco, San Francisco, CA 94143-0412, U.S.A. Tel: 415476 8131; Fax: 415476 0527. nisha.acharya@ucsf.edu.

None of the authors have any financial disclosures related to the subject material in this manuscript. 
oligoarticular ( 4 or less joints involved during first 6 months then 5 or more joints involved thereafter), rheumatoid-factor-positive polyarticular and rheumatoid-factor-negative polyarticular. The incidence of JIA in US and Canada is estimated at 0.041 to 0.061 per 1000 children [3]. Thirty to sixty percent of all JIA in the US and Europe is oligoarticular, which has a peak onset of 2-4 years of age while polyarticular JIA has a bimodal onset, the first at 1-4 years of age and the second at 6-12 years. The female: male ratio in oligoarticular JIA is 3:1[3].

Susceptibility to JIA is inherited; siblings of patients with JIA have a 15-30 fold higher risk of developing JIA compared to the general population. Evidence suggests that JIA is largely a T-cell mediated disease. Dysregulation of cytokines, especially tumor necrosis factor (TNF), contributes to the etiology in both oligoarticular and polyarticular JIA. Environmental triggers are hypothesized but remain unsubstantiated [3].

\section{Ocular Manifestations of JIA}

A large, population based study of JIA patients in Germany found a $12 \%$ prevalence of uveitis in all types of JIA, including $25 \%$ in oligoarticular extended and $16 \%$ in oligoarticular persistent [4]. The mean age of uveitis presentation was 5.2 years. Eightythree percent of patients with uveitis had anterior uveitis, followed by $9 \%$ with intermediate uveitis, $1 \%$ posterior uveitis and $7 \%$ panuveitis. Seventy percent of uveitis patients had bilateral involvement. Predictors of complications were presence of complications at first visit and presentation of uveitis before arthritis, but not ANA positivity.

Retrospective series have reported that $28-67 \%$ of patients with JIA-associated uveitis develop ocular complications [5, 6]. In one series, $12 \%$ had a poor visual outcome [5] and in another, $36 \%$ of affected eyes had 20/50 or worse and $24 \%$ had 20/200 or worse visual acuity at presentation [6]. The main causes of poor vision were cataract, band keratopathy and glaucoma.[6] Active intraocular inflammation ( $\geq 0.5+$ cells) was associated with increased risk of visual impairment and blindness.

A recent retrospective review by Saurenmann et al [7] found the rate of uveitis was significantly higher in girls aged 1-2 years (37\%), compared to the rate of $7 \%$ in boys in the same age group. The same relationship held true in ANA positive patients aged 1-2 years, with $47 \%$ of girls and $10 \%$ of boys having uveitis. The rate decreased to $<10 \%$ in girls with onset of JIA > 7 years of age. In a recent retrospective review of 117 eyes (65 patients), male gender was independently associated with need for cataract surgery, cystoid macular edema, and papillitis and had significantly more of these features at five years follow-up [8] Male gender was associated with a 6.61-fold increased odds of blindness [9].

Thorne et al recently retrospectively examined 75 consecutive patients (132 eyes) with JIAassociated chronic uveitis for the risk of development of cataract. [10].** Significant risk factors included presence of posterior synechiae, active uveitis and topical corticosteroid use at presentation. The overall incidence of new-onset cataract over a median follow-up of 4 years was $0.04 / \mathrm{EY}$. The incidence of new cataract was $0 / \mathrm{EY}$ among patients receiving $\leq 2$ drops of topical corticosteroids per day and 0.16/EY among patients receiving $>3$ drops daily.

\section{Medical Treatment of JIA and JIA-associated uveitis}

The use of biologics in JIA has recently been reviewed [11, 12]. FDA-approved agents for treating JIA are adalimumab [13] and etanercept, both TNF-alpha blockers, and abatacept [14], a T cell activation blocker. By 21 months follow-up, $90 \%$ of patients taking abatacept experienced $>30 \%$ improvement in JIA, as defined by the American College of 
Rheumatology Pediatric 30 Criteria [15]. In contrast, a randomized controlled trial of infliximab $3 \mathrm{mg} / \mathrm{kg}$ plus methotrexate for children with polyarticular JIA did not show a statistically significant difference compared to placebo [16]. The study was instructive in finding that the $6 \mathrm{mg} / \mathrm{kg}$ dose may be safer in children than $3 \mathrm{mg} / \mathrm{kg}$, with fewer serious adverse events, infusion reactions, and development of antibodies. At four years of followup, $44 \%$ achieved improvement of $>30 \%$ in JIA [17].

The mainstay of initial treatment for uveitis remains corticosteroids. For patients requiring chronic therapy, the initial agents of choice are typically antimetabolites including methotrexate, mycophenolate mofetil and azathioprine. Cyclosporine has limited value in the treatment of JIA-associated uveitis [18]. In cases of refractory inflammation, biologics are used, usually in conjunction with an antimetabolite. There are no prospective controlled studies on the use of immunosuppressive medications in children with uveitis. An excellent comprehensive review of the current medical treatment of uveitis in children has recently been published [19]. Use of immunosuppressive therapy has been shown in a retrospective study to be associated with a 74\% reduction in the development of hypotony $(P=0.002)$, $86 \%$ reduction in the development of epiretinal membrane $(P=0.05)$ and $60 \%$ reduction in the development of 20/200 or worse visual acuity in the better eye $(P=0.04)$ [2].

Randomized clinical trials are currently underway to investigate the effectiveness of TNFalpha inhibitors for JIA-associated uveitis. The Multi Center Prospective Registry of Infliximab Use for Childhood Uveitis is an open label Phase IV clinical trial (ClinicalTrials.gov Identifier NCT00589628, accessed June 21, 2010) randomizing children age 4-18 with JIA-associated persistent uveitis to monthly infusions of infliximab of either $5 \mathrm{mg} / \mathrm{kg}$ or $10 \mathrm{mg} / \mathrm{kg}$, with up to 10 months of follow-up. Estimated enrollment is 40 patients. A pilot study of 12 children randomized to either etanercept or placebo for six months followed by open label use of etanercept in all 12 patients for another six months did not find a significant difference between etanercept and placebo in control of uveitis [20]. A larger scale open label Phase III clinical trial (ClinicalTrials.gov Identifier NCT00012506, accessed June 21,2010 ) is currently underway in children age $2-18$ on etanercept for JIAassociated uveitis.

There have also been case series on other biologic drugs and JIA-associated uveitis.

Abatacept has been reported to control refractory uveitis in a girl with psoriatic arthritis and IgA deficiency [21]. A case series reported remission of refractory JIA-associated uveitis in 6 of 7 seven patients taking abatacept after a mean of 9.2 months of treatment [22]. Other biologics in investigation include IL-1 antagonists such as anakinra and the IL-6 antagonist tocilizumab [12]. High-dose daclizumab, a humanized monoclonal antibody against IL-2 receptor, has been reported to provide a two-step reduction in anterior chamber cells in 4 of 6 children with JIA uveitis [23]. However, the manufacturer of daclizumab discontinued production in the US in September 2009, citing declining market demand.

As more patients receive immunosuppressive therapy for ocular inflammatory disease, the long-term safety profile of these treatments becomes important, particularly in children with JIA, who may require systemic treatment for decades. A meta-analysis of clinical trials in which patients with adult rheumatoid arthritis randomized to TNF alpha inhibitors with 2254 week follow-up found a 3.3 fold increase in the risk of cancer [24]. A recent large scale retrospective cohort study by Kempen et al included 7957 US residents with non-infectious ocular inflammatory disease of whom 2340 received immunosuppressive therapy [25]*. Patients who took antimetabolites, $T$ cell inhibitors, and corticosteroids had overall and cancer-related mortality similar to patients who never took immunosuppressive medications and similar to the general US population. Patients who took TNF-alpha inhibitors had increased overall and cancer-related mortality. Even though this result raises concern for the 
safety of TNF inhibitors, the sample size and person-year follow-up of the TNF group was only a fraction of the total study population, reflecting the study enrollment end date in 2005. The US Food and Drug Administration has issued a black box warning on the increased risk of cancer, in particular lymphoma, in children and adolescents taking TNF alpha inhibitors. (www.fda.gov/NewsEvents.Cancer Warnings Required for TNF Blockers.Aug 4 2009.) Even though there was no mention of patients with uveitis, the warning still raises serious concerns.

\section{Surgical management of JIA-associated uveitis}

Successful surgical intervention in the setting of chronic uveitis requires careful planning for optimal results.

\section{Cataract surgery}

Cataract surgery in the setting of uveitis has recently been well reviewed [26, 27].

Historically, authorities have cautioned against primary placement of intraocular lens (IOLs) in patients with JIA because of the risk of secondary cyclitic membranes and development of hypotony [28-30]. With more widespread practice of strict control of uveitis at least 3 months prior to surgery and using systemic immunomodulatory therapy, good postoperative results can be achieved [31]. Even so, the presence of an IOL can still incite recalcitrant inflammation, necessitating removal of the implant [32]. Alternatively, pars plana lensectomy with anterior vitrectomy and subsequent aphakia can be an effective way to manage uveitic cataract [33]. Pars plana vitrectomy (PPV) in combination with phacoemulsification has been reported to successfully improve vision in adults and children with chronic uveitis [34-36].

A recent retrospective study by Sijssens et al examined long-term complications after cataract surgery in aphakic and pseudophakic eyes of children with JIA-associated uveitis [37]*. Intraocular inflammation was under "maximal control" for a minimum of three months prior to surgery. There was no difference in perioperative corticosteroid management between the two groups. Nineteen aphakic eyes and 29 pseudophakic eyes (of which 5 had polymethylmethacrylate IOLs and 24 had acrylic) were included in the study. There was no statistically significant difference in complications between the two groups. Cystoid macular edema was higher in the aphakic group, but did not achieve statistical significance. Visual acuity was significantly better in pseudophakic eyes than aphakic eyes up to 7 years after cataract extraction. The findings suggest that IOLs in children with JIAassociated uveitis are neither a risk factor nor protective of development of secondary inflammatory glaucoma. The authors offered the following relative contraindications for IOL placement: age $<4$ years, hypotony, fellow eye IOL-related complication and shallow anterior chamber.

\section{Fluocinolone acetonide (FA) implant}

The intravitreal FA implant was approved in 2005 for chronic noninfectious uveitis affecting the posterior segment (posterior, intermediate and panuveitis). The use of the implant in children and in particular in children with JIA-associated uveitis has not been reported, as the majority of intraocular inflammation in JIA is anterior uveitis. A dose-randomized, historically controlled multicenter trial of the FA implant for patients with recurrent noninfectious posterior uveitis included patients age 6 or older but there was no mention specifically of children as a subgroup [38, 39]. The Multicenter Uveitis Steroid Treatment Trial (MUST) is a randomized double-masked trial comparing the FA implant versus conventional medical therapy for the treatment of chronic noninfectious uveitis in patients 
age 13 and over [40]. Five patients (2\%) had JIA-associated uveitis. Enrollment is complete and results are expected in 2011.

\section{Pars plana vitrectomy}

PPV is variably performed in children with JIA-associated uveitis. It has been advocated historically in recalcitrant chronic uveitis with posterior involvement, prior to widespread use of immunomodulatory medications [41]. Recently, Giuliari et al reported on the results of PPV in 28 eyes of 20 patients ages 16 or younger with more than 6 months postoperative follow-up, including 5 eyes with JIA-associated iridocyclitis [42]. The mean age at time of PPV was 11.2 years. The mean time of follow-up was 13.5 months. All eyes had active intraocular inflammation with or without medical treatment prior to PPV. Surgery was able to reduce systemic medication for control of uveitis. Ninety-six percent of patients had inactive uveitis with or without medical treatment at last postoperative follow-up.

\section{Long-term follow-up of patients with JIA}

The ocular morbidity of JIA-associated uveitis continues well into adulthood. Skarin and colleagues [43] recently reported on the 24-year follow-up of a cohort of 55 consecutive patients and found that $49 \%$ still had active uveitis. Twenty-two percent had uveitic glaucoma and $51 \%$ had cataract at 24 year follow-up. In another study $26.7 \%$ of patients having JIA-associated uveitis $>15$ years duration had a visual acuity $6 / 12$ or worse [44].

\section{Discontinuing Medical Treatment}

With better methods of achieving control of both uveitis and arthritis in patients with JIA, coupled with concerns about the long-term safety profile of immunosuppressive agents, the issue of if and when to stop medical treatment has now become increasingly relevant. The general expert opinion among uveitis specialists is to try to taper immunomodulatory therapy after 12-24 months of quiescence of uveitis. To date, however, there are no studies in the ophthalmic literature addressing when to withdraw treatment. Recently, Foell and colleagues [45] for the Pediatric Rheumatology International Trials Organization (PRINTO) conducted a prospective, open, multi-center, multi-national clinical trial on 364 patients with JIA randomized to withdrawal of methotrexate at either 6 months or 12 months after confirmed clinical remission of JIA (including no active uveitis) while on medical treatment (maximum dose $15 \mathrm{mg} / \mathrm{m}^{2}$ per week). The relapse rate within 24 months of study enrollment was $56.7 \%$ in the 6 -month group and $55.6 \%$ in the 12 -month group. The median time to relapse was 21.0 months in the 6-month group and 23.0 months in the 12-month group. Since JIA joint disease and JIA uveitis follow different disease courses, this study offers little guidance in the timing of withdrawal of medical treatment in JIA uveitis. Further study is needed in this area.

\section{Conclusion/Recommendation}

JIA remains a challenging disease to treat, with ocular morbidity lasting well into adulthood. Randomized controlled trials are needed to assess the efficacy of antimetabolites, as these remain the first-line treatment for chronic JIA-associated uveitis. Long-term safety of biologic treatment is a concern, especially since children with JIA require years of treatment.

\section{Acknowledgments}

Dr. Acharya is supported by a National Eye Institute K23EY017897 grant and a Research to Prevent Blindness Career Development Award. The Department of Ophthalmology at UCSF is supported by a core grant from the National Eye Institute, EY02162. 


\section{References}

1. Ravelli A, Felici E, Magni-Manzoni S, et al. Patients with antinuclear antibody-positive juvenile idiopathic arthritis constitute a homogeneous subgroup irrespective of the course of joint disease. Arthritis Rheum. 2005 Mar; 52(3):826-32. [PubMed: 15751057]

2. Thorne JE, Woreta F, Kedhar SR, et al. Juvenile idiopathic arthritis-associated uveitis: incidence of ocular complications and visual acuity loss. Am J Ophthalmol. 2007 May; 143(5):840-6. [PubMed: 17362866]

3. Macaubas C, Nguyen K, Milojevic D, et al. Oligoarticular and polyarticular JIA: epidemiology and pathogenesis. Nat Rev Rheumatol. 2009 Nov; 5(11):616-26. [PubMed: 19806151]

4. Heiligenhaus A, Niewerth M, Ganser G, et al. Prevalence and complications of uveitis in juvenile idiopathic arthritis in a population-based nation-wide study in Germany: suggested modification of the current screening guidelines. Rheumatology (Oxford). 2007 Jun; 46(6):1015-9. [PubMed: 17403710]

5. Asproudis I, Felekis T, Tsanou E, et al. Juvenile idiopathic arthritis-associated uveitis: Data from a region in western Greece. Clin Ophthalmol. 2010; 4:343-7. [PubMed: 20463803]

6. Woreta F, Thorne JE, Jabs DA, et al. Risk factors for ocular complications and poor visual acuity at presentation among patients with uveitis associated with juvenile idiopathic arthritis. Am J Ophthalmol. 2007 Apr; 143(4):647-55. [PubMed: 17224116]

7. Saurenmann RK, Levin AV, Feldman BM, et al. Risk factors for development of uveitis differ between girls and boys with juvenile idiopathic arthritis. Arthritis Rheum. 2010 Feb 22; 62(6): 1824-8. [PubMed: 20178126]

8. Ayuso VK, Ten Cate HA, van der Does P, et al. Male Gender as a Risk Factor for Complications in Uveitis Associated with Juvenile Idiopathic Arthritis. Am J Ophthalmol. 2010; 149(6):994-9. [PubMed: 20510688]

9. Ayuso VK, Ten Cate HA, van der Does P, et al. Male gender and poor visual outcome in uveitis associated with juvenile idiopathic arthritis. Am J Ophthalmol. 2010 Jun; 149(6):987-93. [PubMed: 20417926]

10*. Thorne JE, Woreta FA, Dunn JP, et al. Risk of Cataract Development among Children with Juvenile Idiopathic Arthritis-Related Uveitis Treated with Topical Corticosteroids. Ophthalmology. 2010 Apr 2. (Epub 4/2/2010). This is a retrospective review that utlitlized a longitudinal data analysis. Even though use of topical corticosteroids was an independent risk factor for development of cataract, the risk of new onset cataract in patients using two drops a day or less was 0/eye-year and the risk was $0.16 / \mathrm{EY}$ in patients using more than three drops a day.

11. Ilowite NT. Update on biologics in juvenile idiopathic arthritis. Curr Opin Rheumatol. 2008 Sep; 20(5):613-8. [PubMed: 18698187]

12. Hayward K, Wallace CA. Recent developments in anti-rheumatic drugs in pediatrics: treatment of juvenile idiopathic arthritis. Arthritis Res Ther. 2009; 11(1):216. [PubMed: 19291269]

13. Lovell DJ, Ruperto N, Goodman S, et al. Adalimumab with or without methotrexate in juvenile rheumatoid arthritis. N Engl J Med. 2008 Aug 21; 359(8):810-20. [PubMed: 18716298]

14. Ruperto N, Lovell DJ, Quartier P, et al. Abatacept in children with juvenile idiopathic arthritis: a randomised, double-blind, placebo-controlled withdrawal trial. Lancet. 2008 Aug 2; 372(9636): 383-91. [PubMed: 18632147]

15. Ruperto N, Lovell DJ, Quartier P, et al. Long-term safety and efficacy of abatacept in children with juvenile idiopathic arthritis. Arthritis Rheum. 2010 Jun; 62(6):1792-802. [PubMed: 20191582]

16. Ruperto N, Lovell DJ, Cuttica R, et al. A randomized, placebo-controlled trial of infliximab plus methotrexate for the treatment of polyarticular-course juvenile rheumatoid arthritis. Arthritis Rheum. 2007 Sep; 56(9):3096-106. [PubMed: 17763439]

17. Ruperto N, Lovell DJ, Cuttica R, et al. Long-term efficacy and safety of infliximab plus methotrexate for the treatment of polyarticular-course juvenile rheumatoid arthritis: findings from an open-label treatment extension. Ann Rheum Dis. 2010 Apr; 69(4):718-22. [PubMed: 20237125] 
18. Tappeiner C, Roesel M, Heinz C, et al. Limited value of cyclosporine A for the treatment of patients with uveitis associated with juvenile idiopathic arthritis. Eye (Lond). 2009 May; 23(5): 1192-8. [PubMed: 18551142]

19. Simonini G, Cantarini L, Bresci C, et al. Current therapeutic approaches to autoimmune chronic uveitis in children. Autoimmun Rev. 2010 May 26. (Epub 6/18/2010).

20. Smith JA, Thompson DJ, Whitcup SM, et al. A randomized, placebo-controlled, double-masked clinical trial of etanercept for the treatment of uveitis associated with juvenile idiopathic arthritis. Arthritis Rheum. 2005 Feb 15; 53(1):18-23. [PubMed: 15696578]

21. Angeles-Han S, Flynn T, Lehman T. Abatacept for refractory juvenile idiopathic arthritisassociated uveitis-a case report. J Rheumatol. 2008 Sep; 35(9):1897-8. [PubMed: 18785302]

22. Zulian F, Balzarin M, Falcini F, et al. Abatacept for severe anti-tumor necrosis factor alpha refractory juvenile idiopathic arthritis-related uveitis. Arthritis Care Res (Hoboken). 2010 Jun; 62(6):821-5. [PubMed: 20191477]

23. Sen HN, Levy-Clarke G, Faia LJ, et al. High-dose daclizumab for the treatment of juvenile idiopathic arthritis-associated active anterior uveitis. Am J Ophthalmol. 2009 Nov; 148(5):696703. e1. [PubMed: 19664754]

24. Bongartz T, Sutton AJ, Sweeting MJ, et al. Anti-TNF antibody therapy in rheumatoid arthritis and the risk of serious infections and malignancies: systematic review and meta-analysis of rare harmful effects in randomized controlled trials. JAMA. 2006 May 17; 295(19):2275-85. [PubMed: 16705109]

25*. Kempen JH, Daniel E, Dunn JP, et al. Overall and cancer related mortality among patients with ocular inflammation treated with immunosuppressive drugs: retrospective cohort study. BMJ. 2009; 339:b2480. This large scale retrospective study reviewed patients with ocular inflammatory disease who took immunosuppressive medications compared with those who did not. Conventional therapy such as corticosteroids, antimetabolites, $\mathrm{T}$ cell inhibitors were not associated with increased overall or cancer-related mortality. Tumor necrosis factor antagonists, however, were associated with increased risk of both overall and cancer-related mortality, even though this subgroup waslimited in the number of patients. [PubMed: 19578087]

26. Jancevski M, Foster CS. Cataracts and uveitis. Curr Opin Ophthalmol. 2010 Jan; 21(1):10-4. [PubMed: 19829114]

27. Van Gelder RN, Leveque TK. Cataract surgery in the setting of uveitis. Curr Opin Ophthalmol. 2009 Jan; 20(1):42-5. [PubMed: 19077828]

28. Holland GN. Intraocular lens implantation in patients with juvenile rheumatoid arthritis-associated uveitis: an unresolved management issue. Am J Ophthalmol. 1996 Aug; 122(2):255-7. [PubMed: 8694094]

29. Probst LE, Holland EJ. Intraocular lens implantation in patients with juvenile rheumatoid arthritis. Am J Ophthalmol. 1996 Aug; 122(2):161-70. [PubMed: 8694084]

30. BenEzra D, Cohen E. Cataract surgery in children with chronic uveitis. Ophthalmology. $2000 \mathrm{Jul}$; 107(7):1255-60. [PubMed: 10889094]

31. Quinones K, Cervantes-Castaneda RA, Hynes AY, et al. Outcomes of cataract surgery in children with chronic uveitis. J Cataract Refract Surg. 2009 Apr; 35(4):725-31. [PubMed: 19304096]

32. Adan A, Gris O, Pelegrin L, et al. Explantation of intraocular lenses in children with juvenile idiopathic arthritis-associated uveitis. J Cataract Refract Surg. 2009 Mar; 35(3):603-5. [PubMed: 19251158]

33. Verbraeken H. Pars plana lensectomy in cases of cataract with juvenile chronic uveitis. Graefes Arch Clin Exp Ophthalmol. 1996 Oct; 234(10):618-22. [PubMed: 8897053]

34. Androudi S, Ahmed M, Fiore T, et al. Combined pars plana vitrectomy and phacoemulsification to restore visual acuity in patients with chronic uveitis. J Cataract Refract Surg. 2005 Mar; 31(3): 472-8. [PubMed: 15811733]

35. Soheilian M, Mirdehghan SA, Peyman GA. Sutureless combined 25-gauge vitrectomy, phacoemulsification, and posterior chamber intraocular lens implantation for management of uveitic cataract associated with posterior segment disease. Retina. 2008 Jul-Aug; 28(7):941-6. [PubMed: 18698295] 
36. Acevedo S, Quinones K, Rao V, et al. Cataract surgery in children with juvenile idiopathic arthritis associated uveitis. Int Ophthalmol Clin. 2008 Spring;48(2):1-7. [PubMed: 18427255]

37*. Sijssens KM, Los LI, Rothova A, et al. Long-term ocular complications in aphakic versus pseudophakic eyes of children with juvenile idiopathic arthritis-associated uveitis. Br J Ophthalmol. 2010 Jun 16. (Epub 6/19/2010). This retrospective series compared long-term outcome of pseudophakic and aphakic eyes of children with JIA-associated uveitis. There was no difference in complications between the two groups. Pseudophakic eyes had better visual acuity compared to aphakic eyes. Pseudophakia was neither protective nor a risk factor for secondary inflammatory glaucoma.

38. Jaffe GJ, Martin D, Callanan D, et al. Fluocinolone acetonide implant (Retisert) for noninfectious posterior uveitis: thirty-four-week results of a multicenter randomized clinical study. Ophthalmology. 2006 Jun; 113(6):1020-7. [PubMed: 16690128]

39. Callanan DG, Jaffe GJ, Martin DF, et al. Treatment of posterior uveitis with a fluocinolone acetonide implant: three-year clinical trial results. Arch Ophthalmol. 2008 Sep; 126(9):1191-201. [PubMed: 18779477]

40. Kempen JH, Altaweel MM, Holbrook JT, et al. The multicenter uveitis steroid treatment trial: rationale, design, and baseline characteristics. Am J Ophthalmol. 2010 Apr; 149(4):550-61. e10. [PubMed: 20097325]

41. Verbraeken H. Therapeutic pars plana vitrectomy for chronic uveitis: a retrospective study of the long-term results. Graefes Arch Clin Exp Ophthalmol. 1996 May; 234(5):288-93. [PubMed: 8740248]

42. Giuliari GP, Chang PY, Thakuria P, et al. Pars plana vitrectomy in the management of paediatric uveitis: the Massachusetts eye research and surgery Institution experience. Eye (Lond). 2010 Jan; 24(1):7-13. [PubMed: 20057512]

43. Skarin A, Elborgh R, Edlund E, et al. Long-term follow-up of patients with uveitis associated with juvenile idiopathic arthritis: a cohort study. Ocul Immunol Inflamm. 2009 Mar-Apr; 17(2):104-8. [PubMed: 19412872]

44. Camuglia JE, Whitford CL, Hall AJ. Juvenile idiopathic arthritis associated uveitis in adults: a case series. Ocul Immunol Inflamm. 2009 Sep-Oct; 17(5):330-4. [PubMed: 19831566]

45. Foell D, Wulffraat N, Wedderburn LR, et al. Methotrexate withdrawal at 6 vs 12 months in juvenile idiopathic arthritis in remission: a randomized clinical trial. JAMA. 2010 Apr 7; 303(13): 1266-73. [PubMed: 20371785] 